\title{
Computational biomodelling and numerical analysis as means of diagnostic and odontological prognosis
}

\begin{abstract}
In routine clinical practice, the dentist performs procedures that include not only the diagnosis of various oral pathologies, but also the treatment of the patient. The Dentist uses his knowledge and technical skills, following all the dogmas and criteria established for the successful rehabilitation of the patient. However, on some occasions and despite all the treatment fails, which generates disagreement on the part of the patient and loss of confidence in the dentist-patient relationship. That is why it must apply means that allow better diagnoses and that contribute to a successful treatment. The biomodels can be tools that allow from the diagnosis to the prediction of the treatments applied.
\end{abstract}

Volume 2 Issue 4 - 2018

\author{
Rodrigo Arturo Marquet Rivera, Guillermo \\ Urriolagoitia Sosa, Rosa Alicia Hernández \\ Vázquez, Beatriz Romero Ángeles, Juan \\ Alejandro Vázquez Feijoo, Guillermo \\ Urriolagoitia-Calderón \\ Instituto Politécnico Nacional, México
}

Correspondence: Guillermo Urriolagoitia Sosa, Instituto Politécnico Nacional, Escuela Superior de Ingeniería Mecánica y Eléctrica, Sección de Estudios de Posgrado e Investigación Unidad Profesional Adolfo López Mateos, Zacatenco. Av. IPN s/n Edificio 5, $2^{\circ}$ piso Col. Lindavista, Delegación Gustavo A. Madero, C.P. 07320 , México, Email guiurri@hotmail.com

Received: June 30, 2018 | Published: August 21, 2018

\section{Introduction}

The failure of the dental treatment can be due to multifactorial situations. Nevertheless, most of the time they are the result of situations inherent to the patient. ${ }^{1}$ The Dentist must then look for the tools that allow him to find these situations. The computational biomodels of the dental organs and their analysis by means of the Finite Element Method (MEF) are this useful tool that can be used, in a personalized way for the cases of patients who present this situation. $^{2}$ Although a further study of the patient could increase the initial budget of the treatment, it also means avoiding a sequence of treatments that are applied to the trial that would increase the budget even more, as well as the patient's disagreement. The elaboration of biomodels and their analysis, although laborious is a tool that would help find the origin of the treatment failure, provide a better treatment and correct it in a shorter time and with greater patient satisfaction.

\section{Proposal}

The biomodelling of dental organs and their numerical analysis is mostly used for research. This is due to its precision and approach to reality, it is also possible to use it as a clinical tool by the Dentist in his private practice. With it, is possible offer at patients better and more appropriate treatments and at the same time their satisfaction will be greater. First, with computer models it is possible to establish better diagnoses. The diagnostic methods used in Dentistry mainly include those that are applied directly at the time of consultation and secondly those obtained through digital imaging. Unfortunately, the programs or viewers that offer the radiological services only allow a very reduced vision to explore the structures of the Stomatognathic System. This greatly limits the visualization of some sections. With biomodels, it is possible to see more clearly and accurately diagnostic signs with greater objectivity (Figure 1).

With biomodels, it is possible to find different agents without direct intervention. In figure 1, the findings found were not visible with an $\mathrm{x}$-ray or the tomography with the naked eye, since the restoration and the enamel itself did not allow its visualization. The biomodel allows manipulation to virtually remove the restoration and the enamel itself. Likewise, it allows to establish the prognosis of the treatments. In Figure 2, through the biomodel, the critical points of a restoration can be observed due to lack of sealing and adjustment. With this, it is possible to intervene before ending the treatment and give a better prognosis, as well as providing a successful treatment. The numerical analyzes will contribute to the prediction of the aforementioned critical zones (Figure 2).
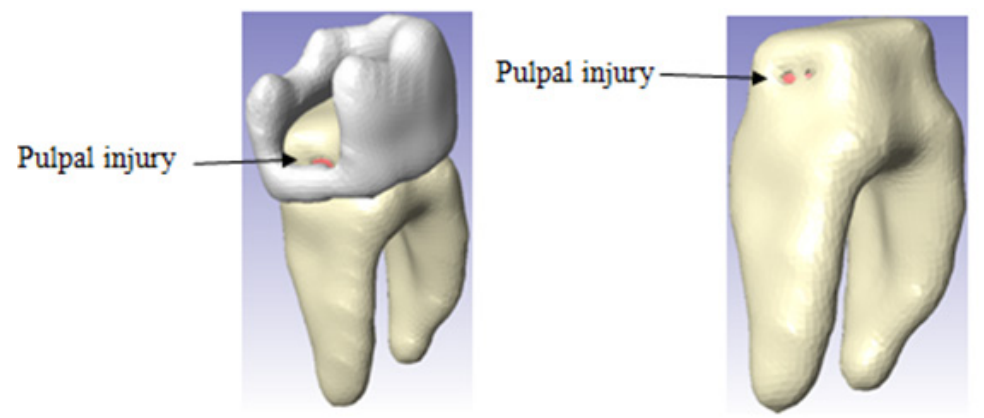

Figure I Computational biomodels of a restored molar.Through the biomodel, the pulpal damage was found after the restoration of the same. 
a)

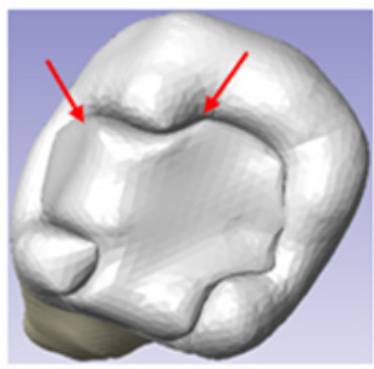

b)
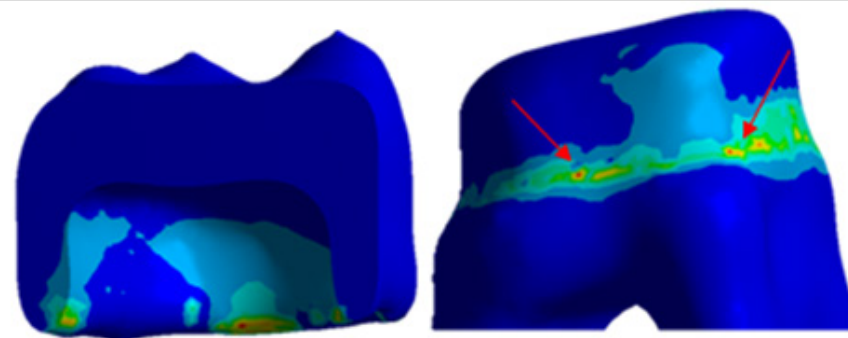

Figure 2 Biomodels for treatment prognosis: a) Mismatch zones, b) Critical areas.

\section{Discussion and conclusion}

The numerical analysis as well as the use of biomodels, should be known in the area of Dentistry as powerful tools that can be used as diagnostic means and even to achieve the manufacture of restorations through CAM / CAD programs and 3D printing. Nonetheless, its management is not the general domain of the medical or dental community. Therefore, it is necessary to disseminate this knowledge through multidisciplinary working groups, which will allow a better treatment to patients from the dental office. Although these computer tools are not totally unknown in the area of Odontology, their use has not been exploited to their full potential. It is considered that its use is indicated only for research. The Dental Surgeon can use these means to facilitate the diagnosis and be able to perform treatments with greater precision. In addition, they turn out to be much more innovative means which will improve their practice and patient satisfaction.

\section{Acknowledgments}

The authors thank the Instituto Politécnico Nacional and the Consejo Nacional de Ciencia y Tecnología for the support provided, in the preparation of this work.

\section{Conflict of interest}

Author declares that there is no conflict of interest.

\section{References}

1. Otero Injoque J. Gestión del fracaso del tratamiento dental. Revista Cientifica Odontológica de la Universidad Científica del Sur. 2016;4(2):593-596.

2. Wakabayashi N, Ona M, Suzuki T, et al. Nonlinear finite element analyses: advances and challenges in dental applications. $J$ Dent. 2008;36(7):463471. 